\title{
Estudio de la variabilidad de la precipitación y la temperatura en la cuenca del Río Quindío
}

\section{Study of the variability of the precipitation and temperature in the Quindio River basin}

\author{
Pedro León García Reinoso*, Elkin Aníbal Monsalve Durango,* César Augusto Bustamante Toro**
}

Recibido: Abril 23 de 2009

Aceptado: Junio 25 de 2009

Correspondencia: Programa de Ingeniería Civil, Universidad del Quindío. Avenida Bolivar calle 12 norte Armenia Quindío. Email. pedrogarcia@uniquindio.edu.co

\section{RESUMEN}

Las dificultades entorno a la calidad y cantidad del agua evidencian que hacia el 2025 el $41 \%$ de la población no contará con disponibilidad de agua para consumo y, donde la principal fuente hídrica, el Río Quindío, tiene comprometido el $80.4 \%$ de su producción. La quema de combustibles fósiles incrementa la concentración de gases de efecto invernadero en la atmósfera. Se estima que estas alteraciones cambiarán el clima regional y global, así como los parámetros relacionados con éste. El objetivo principal del estudio fue establecer una perspectiva de las tendencias de las variables climatológicas en la cuenca del Rió Quindío, seleccionando estaciones que contengan registros de temperatura y precipitación, como los elementos climáticos que mejor describen el comportamiento del clima en el componente atmosférico. Se propone identificar el comportamiento de las variables seleccionadas, por medio de la construcción de modelos ARIMA, utilizando la metodología de Box and Jenkins (1976). Las series de precipitación no muestran ningún tipo de tendencia, exhiben variaciones que podrían asumirse como estacionaria en su parte regular, el modelo de mejor ajuste fue un $\operatorname{ARIMA}(1,0,0)(2,1,0) 12$. Las series de temperatura mostraron fuertes tendencias de crecimiento y la presencia de periodos atípicos que están relacionados con eventos climáticos anómalos, el modelo de mejor ajuste fue un $\operatorname{ARIMA}(3,1,0)(1,1,0) 12$.

Palabras Clave: Cambio Climático. Hidrología. Modelos Arima.

\begin{abstract}
The difficulties in regard to water quality and quantity make clear that to $2025,41 \%$ of the population will not count with water availability for assumption, and that, the main aquatic source, the Quindio River, has compromised the $80.4 \%$ of its production. The fossil fuels burning increases the green house gasses concentration in the atmosphere. It is estimated that these alterations will change the regional and global climate, and so the parameters related to it. The main objective of the study was to establish a perspective of the climate variable tendencies in the Quindio River Basin, by selecting stations that contain records of temperature and precipitation as the climatic elements that describe the best the water comportment in the atmospheric component. It is proposed to identify the comportment of the selected variables by means of the construction of ARIMA models, using Box and Jenkins' (1976) methodology. The precipitation series do not show any tendency type, they exhibit variations that could be assumed as stationary on their regular part, the best adjust model was an ARIMA $(1,0,0)(2,1,0) 12$. The temperature series showed strong growth tendencies and the presence of atypical periods that are related with anomalous climatic events, the best adjust model was an ARIMA $(3,1,0)(1,1,0) 12$.
\end{abstract}

Key words: Climate Change. Hydrology. ARIMA Models.

\section{INTRODUCCIÓN}

$\mathrm{H}$ ay suficientes pruebas de la variabilidad climática en una amplia gama de escalas temporales en toda América Latina. En muchas subregiones de América Latina, esta variabilidad en el clima normalmente tiene que ver con fenómenos que ya han producido impactos con importantes consecuencias socioeconómicas y medioambientales. El clima está cambiando, atribuido directa o indirectamente a actividades humanas que alteran la composición de la

*Programa de Ingeniería Civil. Facultad de Ingeniería. Universidad del Quindío. Investigadores Grupo CIDERA

** Programa de Licenciatura en Biología y Educación Ambiental. Universidad del Quindío. Investigador Grupo CIDERA 
atmósfera mundial, y que viene a añadirse a la variabilidad natural del clima. Se estima que estas alteraciones cambiarán el clima regional y global, así como los parámetros relacionados con éste. Se han identificado cambios potencialmente graves, como el aumento de la incidencia de fenómenos extremos como altas temperaturas, crecidas y sequías, generando incendios, brotes, plagas y cambios en la composición, estructura y funcionamiento del ecosistema (IPCC, 2003). No queda entonces, sino la obligación de reducir las distancias entre los conocimientos actuales y las necesidades para la toma de decisiones de política a través de la valoración de la información disponible, permitiendo en el corto plazo inferir escenarios con respecto a la sensibilidad, capacidad de adaptación y vulnerabilidad de los sistemas naturales humanos al cambio climático.

Colombia no es ajena a este fenómeno, aunque el país contribuye en una mínima parte al cambio climático, emitiendo únicamente el $0.25 \%$ de las emisiones globales de dióxido de carbono (IDEAM, 2001), no está exento a los efectos que éste le pueda causar. Según la Primera Comunicación Nacional de Colombia sobre el Cambio Climático, se determinó que los ecosistemas colombianos más vulnerables a los efectos del cambio climático serían los de alta montaña. Partiendo de este supuesto, estudios realizados para el Departamento del Quindío, evidencian en torno a la calidad y cantidad de agua que para el año 2025 el $41 \%$ de la población no contaría con disponibilidad de agua para su consumo (CRQ, 2004).

El clima es un sistema complejo por lo que su comportamiento es muy difícil de predecir. Por una parte hay tendencias a largo plazo debidas, normalmente, a variaciones sistemáticas como el aumento de la radiación solar o las variaciones orbitales pero, por otra, existen fluctuaciones caóticas debidas a la interacción entre forzamientos, retroalimentaciones y moderadores.

Desde hace algún tiempo el hombre ha evolucionado en cuanto a tecnología se refiere, creando aparatos de medición más precisos que registran regularmente (diaria, mensual, entre otros) diversas variables meteorológicas. Estos datos se utilizan tanto para la elaboración de predicciones meteorológicas a partir de modelos numéricos como para estudios climáticos.

La Corporación Autónoma Regional del Quindío (CRQ) es la autoridad ambiental en el ámbito local. En el desarrollo de sus funciones se implementó una red hidrometeorológica que inició registros en el año de 1971, complementando la estación Aeropuerto El Edén, que opera desde 1949 y es administrada por el Instituto de Hidrología, Meteorología y
Estudios Ambientales (IDEAM). Sólo hasta el año 2003 se realizó la compilación y el análisis preliminar de la información registrada por la CRQ (Arango, 2003), que permitió la edición del primer Boletín Meteorológico (CRQ, 2003). La publicación fue mejorada por un trabajo posterior aún no publicado (Peláez, 2005). El estudio que hoy se presenta, constituye la primera revisión formal de las series históricas de precipitación y temperatura, con el deseo que sus resultados sean considerados en la construcción de herramientas técnicas que soporten la toma de decisiones en lo que a la gestión del recurso hídrico se refiere.

El propósito principal del trabajo es dar a conocer las tendencias de variables climatológicas en la Cuenca del Río Quindío, basados en los modelos ARIMA (Autorregresivos, Integrados y de Media Móvil) enmarcados en la metodología Box-Jenkins, que procura ajustar el modelo ARIMA utilizando como criterio de selección el residuo y sus parámetros estadísticos, además involucra la estacionalidad de las series de tiempo. La Metodología involucra el desarrollo iterativo de tres fases: Identificación del modelo, selección de parámetros y diagnóstico del modelo.

\section{MATERIALES Y MÉTODOS}

\section{Zona de estudio}

El departamento del Quindío está situado en el centro geográfico del occidente colombiano. La mayor parte del territorio comprende la Hoya del Río Quindío y la vertiente occidental de la cordillera central. Limita: al norte con el departamento de Risaralda desde la desembocadura de la quebrada San Felipe en el río La Vieja hasta la quebrada el Castillo en el Río Quindío; desde éste sitio, por el este y el sur hasta el Páramo de Barragán con el departamento del Tolima; desde este ultimo lugar por el este y el sur hasta la desembocadura de la quebrada San Felipe, con el departamento del Valle, punto de partida.

En la Figura 1 se presenta la cuenca del río Quindío. El cauce principal de esta cuenca nace en el nevado El Quindío (3780 m.s.n.m) al noreste del departamento; en los límites con el departamento del Valle del Cauca recibe las aguas del río Barragán el cual nace en el sur del departamento en el páramo de Barragán, proyectándose desde allí hacia el norte. La convergencia de los ríos Quindío y Barragán da origen al río La Vieja.

La cuenca del río Quindío provee de agua a los acueductos municipales de Armenia, Circasia, Salento y La Tebaida permitiendo el abastecimiento de éste vital líquido aproximadamente a 300.000 habitantes quienes representan el 55\% de la población Quindiana. Además la 
Cuenca del Río Quindío es una zona de amortiguación que potencia, amplía y posibilita el funcionamiento ecológico del Parque Nacional Natural de los Nevados, de hecho, la parte superior de la Cuenca del Río Quindío se encuentra dentro de los límites del Parque de los Nevados.

La cuenca representa un valor de particular importancia para el Quindío en términos productivos, ya que sus singulares escenarios naturales han promovido el ecoturismo cuya visión futura es fuente promisoria de empleo y bienestar (Gobernación del Quindío, 2005). Como ejemplo, el embalse para el acueducto regional esta proyectado en una de sus subcuencas: El Río Navarco.

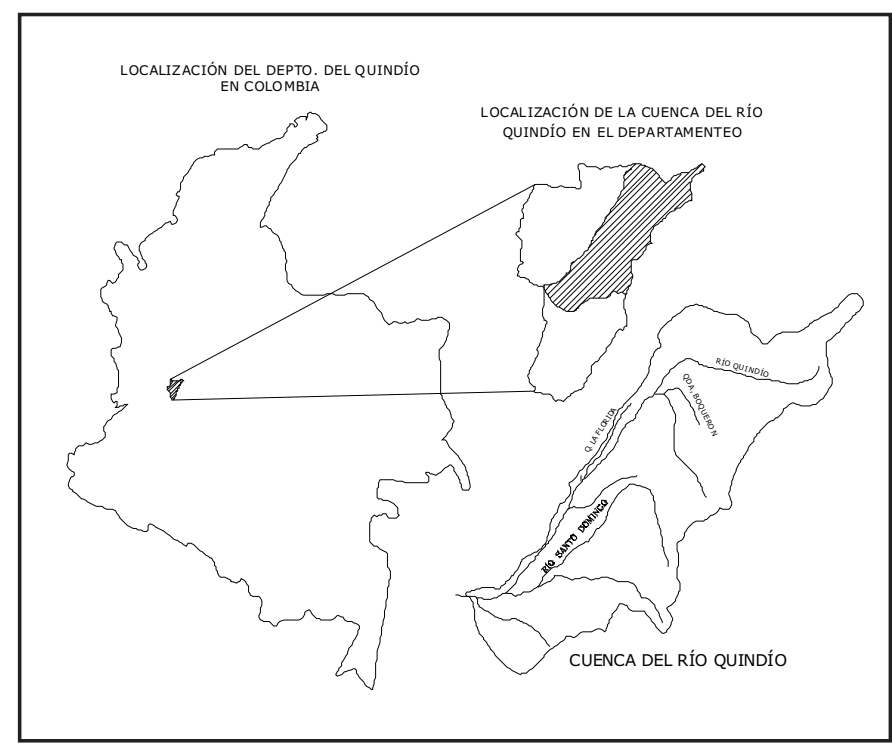

Figura 1 - Cuenca del Río Quindío (Lozano et. al., 2002)

\section{Selección de estaciones}

El estudio se desarrolló con registros mensuales de precipitación ( $\mathrm{P}$ ) y temperatura $(\mathrm{T})$ de estaciones monitoreadas por el Instituto de Hidrología, Meteorología y Estudios Ambientales (IDEAM), el Centro Nacional de Investigaciones del Café (CENICAFE) y la Corporación Regional Autónoma del Quindío (CRQ), que se encuentren dentro y alrededor de la Cuenca del río Quindío, según se muestra en la Figura 2. Dada la existencia de series de brillo solar, se realizó un análisis exploratorio comparativo con la serie de temperatura.

Para asegurar la calidad en el análisis de las series de tiempo se adoptó la selección de aquellas estaciones que contaron con amplios periodos de registro, disponibilidad de parámetros y cercanía al área de estudio. En detalle:

* Periodo de registro: En la práctica un periodo cercano a los 30 años es suficiente para que estén en él incluidas todas las características de los regímenes del elemento climático (García, 2007).
*Variables: La temperatura, la precipitación, el brillo solary la evaporación, son las que mejor describen el comportamiento de la atmósfera. Las estaciones seleccionadas contaron con dos o más variables.

*Cercanía al área de estudio: La Organización Meteorológica Mundial acepta en regiones montañosas una estación por cada $250-1000 \mathrm{~km} 2$. Además de las estaciones localizadas al interior de la cuenca, se consideraron las estaciones administradas por CRQ, IDEAM y CENICAFE, con registros superiores al 95\% del tiempo observado.

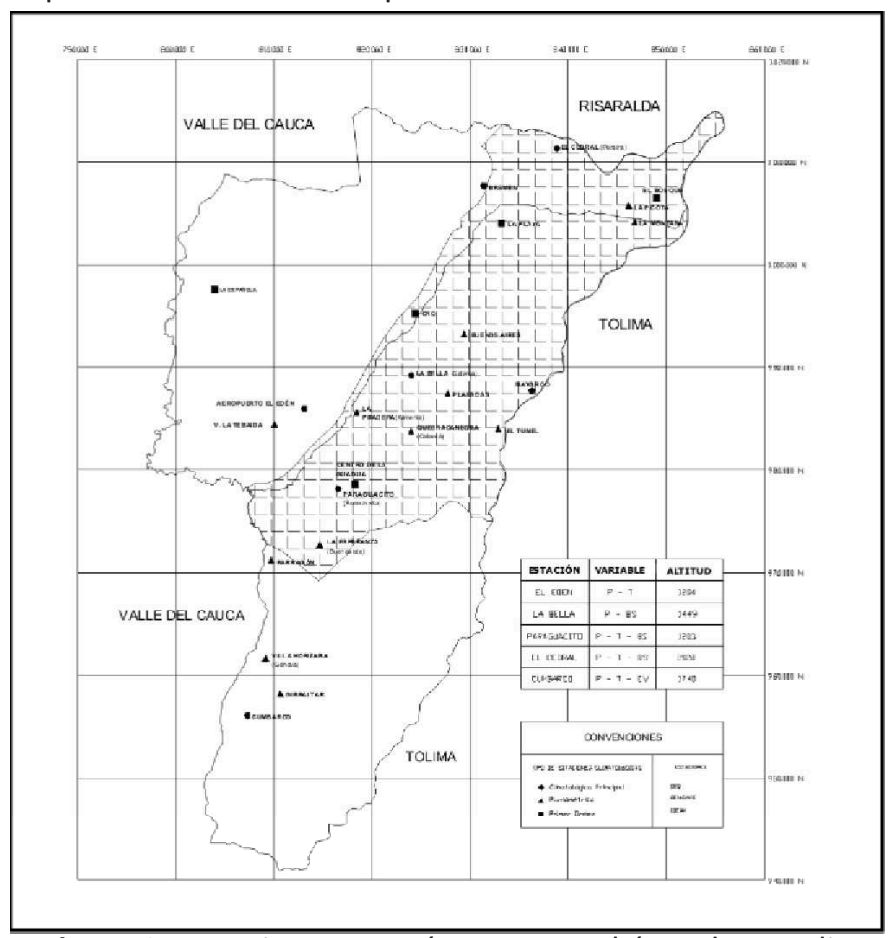

Figura 2 - Estaciones y parámetros en el área de estudio

(Pérez \& Pedraza, 2007)

\section{Análisis exploratorio de datos}

Las graficas de los datos muestran la variabilidad temporal, lo que permite recopilar información preliminar para explorar la existencia de una tendencia en la serie del tiempo (Hurtado y Salcedo, 1996). Para el estudio se incluyeron aspectos de análisis gráfico y modelos estadísticos para la detección de cambios o tendencias con gráficos como: Series Suavizadas, Series Diferenciadas y Correlogramas.

\section{Análisis estocástico}

El análisis estocástico se orientó en la revisión del comportamiento estadístico de las series, para determinar la periodicidad y dependencia de la misma, si existe o no, un componente aleatorio en ellas y determinar la importancia de componentes como el movimiento a largo plazo, la amplitud de las oscilaciones, la presencia de valores atípicos o anómalos, entre otros. Las alternativas de análisis se identifican según los órdenes de los parámetros que se 
se estiman, identificándose:

*Autoregresivos $A R(p)$

* De media móvil MA(q)

*Autoregresivo media móvil ARMA (p,q)

*Autoregresivo integrado de media móvil ARIMA $(p, d, q)$

*Autoregresivo Integrado de Media Móvil Estacional para un proceso estacional con período conocido $\operatorname{SARIMA}(p, d, q)(P, D, Q)$

Para procesos estacionales, el modelo ARIMA se puede expresar como ARIMA(p,d,q)x(P,D,Q)S donde los valores $P, D, Q$ son los órdenes autorregresivo, de diferenciación y del promedio móvil, respectivamente del componente estacional y $\mathrm{s}$ la estacionalidad. Para obtener el modelo de series temporales se siguen los siguientes pasos:

*Ingreso de Datos: Se incorporan los datos mensuales como un vector.

*Gráfica de la serie: Con un graficador se ilustra la serie histórica a fin de decidir la estacionariedad.

*Identificación de tendencias: En el suavizado de las series se consideró el valor observado en el mes y los valores de meses adyacentes, con un procedimiento poco sensible a la presencia de datos extremos. La curva suavizada proporcionó el nivel medio de las variables a analizar, con lo que se identifica de mejor forma la tendencia en la serie de tiempo.

*Diferenciaciones: El procedimiento permite identificar el parámetro $d$ y $D$ en su parte no estacional o estacional. La diferenciación fue necesaria siempre que la media mostró un comportamiento inestable.

*Eliminación de la tendencia: Al observar el gráfico suavizado se identifica la tendencia, posteriormente se remueve.

*Identificación del modelo: Este se logra con el análisis de los correlogramas de las funciones de autocorrelación simple (FAS) y de autocorrelación parcial (FAP) que corresponden a los procesos autoregresivos y de medias móviles de la parte regulary estacional.

*Estimación de los coeficientes del modelo: Como se trata de un proceso iterativo de cálculo, pueden indicarse valores iniciales.

* Contraste de validez del modelo: Se usan procedimientos para evaluar el o los modelos preseleccionados, contraste de significación de parámetros, coeficiente de correlación, etc.
*Análisis de los errores: Las diferencias entre los valores observados y los estimados por el modelo es una fuente de interés para valorarlo.

*Selección del modelo: con los resultados de las etapas anteriores se decide sobre el modelo definitivo.

*Predicción: el modelo seleccionado se usará como fórmula de predicción.

La metodología que se utilizó para el estudio de series de tiempo fue la de Box-Jenkins (1976), que procura ajustar el modelo ARIMA utilizando como criterio de selección el residuo y sus parámetros estadísticos, además involucra la estacionalidad de las series de tiempo, estos autores popularizaron una metodología para encontrar el modelo,ARIMA $(p, d, q)$ SARIMA $(P, D, Q) s$

El procedimiento consta de tres etapas que se aplican de manera iterativa hasta alcanzar un resultado satisfactorio (Tabla 1). Cada etapa se describe a continuación:

\section{1) Identificación del modelo}

El análisis y modelado de datos comúnmente encontrados en recursos hídricos a menudo asume que estos son estacionarios en la media y en la covarianza. Sin embargo, si hay cambios o tendencias en los datos, la suposición de estacionariedad no es válida. En estos casos, el cambio o la tendencia en los datos necesitan ser identificados, modelados, estimados y, en algunos casos, removidos de la serie original para análisis posteriores.

Suponga que se dispone de $\mathrm{n}$ observaciones igualmente espaciadas en el tiempo que corresponden a una realización de un proceso. Se trata de que a partir de esta realización se puedan determinar:

* Las transformaciones que deben hacerse a las variables del proceso para que la varianza sea constante.

*El orden de diferenciación que debe aplicarse a las variables para generar un proceso con media constante.

* La transformación de la serie cuando presenta no estacionariedad en la parte estacional, en tal caso, puede requerirse una diferenciación estacional (D).

* Los componentes autorregresivo y de promedio móvil para relacionar las variables del proceso (hallar p y q en el modelo $\operatorname{ARIMA}(p, d, q))$.

* La identificación de los componentes autorregresivo y de 
Tabla 1 - Fases de la metodología Box - Jenkins (Pérez \& Pedraza, 2007)

\begin{tabular}{|c|c|}
\hline Identificación & $\begin{array}{c}\text { - Recogida de datos } \\
\text { Representación gráfica }\end{array}$ \\
\hline stimación & $\begin{array}{c}\text { Análisis de la hipótesis de estacionariedad (media y varianza) } \\
\text { I Identificación de los procesos regular y estacional }\end{array}$ \\
\hline Diagnóstico o chequeo & $\begin{array}{c}\text { Estimación de los parámetros de los modelos ARIMA candidatos } \\
\text { - Análisis de los residuos }\end{array}$ \\
\hline
\end{tabular}

promedio móvil, esto es los ordenes p y q. Para ello deberá estudiarse simultáneamente el comportamiento de la Función de Autocorrelación Simple (FAS) y la Función de Autocorrelación Parcial (FAP). En un proceso AR(p) la FAS decae geométricamente mientras que la FAP cae rápidamente a cero. Un proceso $\mathrm{MA}(q)$ la FAS cae rápidamente a cero, con solamente las primeras $q$ correlaciones simples significativamente diferentes de cero, mientras que la FAP decae en forma geométrica. En un proceso $\operatorname{ARMA}(p, q)$ ambas funciones tienen un comportamiento geométricamente decreciente a partir de ciertos valores de $k$ así: la FAS comienza su decrecimiento a partir de $k=q+1$ y la FAP a partir de $k=p+1$.

\section{2) Estimación de los parámetros}

Una vez elegidos los órdenes $(p, d, q)$ en la parte regular y $(P, D, Q)$ en la parte estacional se procede a estimar los parámetros Autoregresivos y de Medias móviles que intervienen. Los estimadores del modelo ajustado a la serie estacionaria observada, deben cumplir las condiciones de estacionariedad (la suma de los valores de los coeficientes de cada parámetro deberá ser < |11 y garantizar la independencia de los errores tipo 1, P-valor< 5\%). Se trata de probar la siguiente hipótesis: $\quad$ Ho: $1=2=\ldots=k=0$ (Hipótesis nula) (Hurtado, 1996). Esta característica determina que el procedimiento completo no sea recomendable en situaciones de escasez de datos.

\section{3) Diagnóstico del modelo}

El modelo estimado debe representar adecuadamente el proceso generado por las observaciones. Una estructura adecuada será aquella que cumpla, al menos, las siguientes condiciones:

*Admisibilidad: El modelo estimado es coherente con el conocimiento previo del fenómeno.

*Parametrización: El número de parámetros estimados debe ser lo más reducido posible.
* Coherencia con los datos: La estructura presenta un buen ajuste a las observaciones, los residuos son pequeños y aleatorios (ruido blanco).

En el caso de los modelos ARIMA hay dos cuestiones que revisten especial importancia: Cumplimiento de las condiciones de estacionariedad (la hipótesis base es que el proceso estocástico es estacionario) y el residuo de cualquier modelación debe ser pequeño y errático.

\section{RESULTADOS Y DISCUSIÓN}

\section{Precipitación}

Por registrar el mayor periodo de registro y representar la cuenca media, alta y baja, se presentan los resultados de las Estaciones El Cedral, La Bella y Paraguacito, respectivamente. En la Figura 3, la grafica izquierda corresponde con la serie de tiempo y la derecha muestra el resultado de la proyección.

Se observa como todas las graficas originales de precipitación guardan relación entre ellas, identificándose variaciones en periodos de tiempo iguales, siendo más sobresalientes los años 1970 a 1976 donde ocurren oscilaciones fuertes y prolongadas igual ocurre cerca al año 2000. Estas fluctuaciones podrían estar asociadas a algún tipo de fenómeno climatológico.

Visualmente no hay presencia de algún tipo de tendencia creciente $o$ decreciente y aunque la serie exhibe algunas variaciones se podría asumir como estacionaria en su parte regular. Al observar las gráficas FAS y FAP originales se apreció la presencia de un componente estacional, dando paso a una diferenciación estacional periodo 12.

Se identificó un modelo ARIMA como un proceso autoregresivo AR. Al parecer las estaciones con mayor número de datos en sus registros (La Bella y Aeropuerto El Edén) mostraron un modelo, $\operatorname{ARIMA}(\mathbf{1}, \mathbf{0 , 0})(\mathbf{2}, \mathbf{1 , 0} \mathbf{0} \mathbf{1 2}$ Aquellas con menor periodo de registro presentaron un 
modelo, ARIMA(1,0,0)(1,1,0)12. Todas presentaron buen ajuste en los coeficientes de cada uno de los parámetros, evidenciando este criterio con la incorrelación entre los residuales.

Como apoyo, la presencia gráfica de los horizontes de proyección expresan el buen margen de confiabilidad en datos futuros por ser coherente con el conocimiento previo del fenómeno Se infiere un comportamiento normal sin evidencia de tendencia. Los valores fluctúan alrededor de una media que permanece constante en el tiempo. El error en la proyección se incrementa con el horizonte de predicción.

\section{Temperatura}

En la Figura 4, la grafica izquierda corresponde con la serie de tiempo y la derecha muestra el resultado de la proyección. El
Aeropuerto El Edén es una estación operada por el IDEAM, localizada en una zona plana cuyas condiciones físicas se han mantenido en el tiempo. Los datos que en ella se registran sirven para validar los registros de otras estaciones de la región.

Las series de temperatura mostraron fuertes tendencias de crecimiento notándose un comportamiento similar en la totalidad de las series.

Se presentó de igual forma, la presencia de periodos atípicos que se ven referenciados en las graficas originales al igual que en las suavizadas alrededor de los años 1973 -1976 y 1998 1999, las cuales corresponden a periodos de fuertes oscilaciones que podrían atribuirse a algún evento climático anómalo.
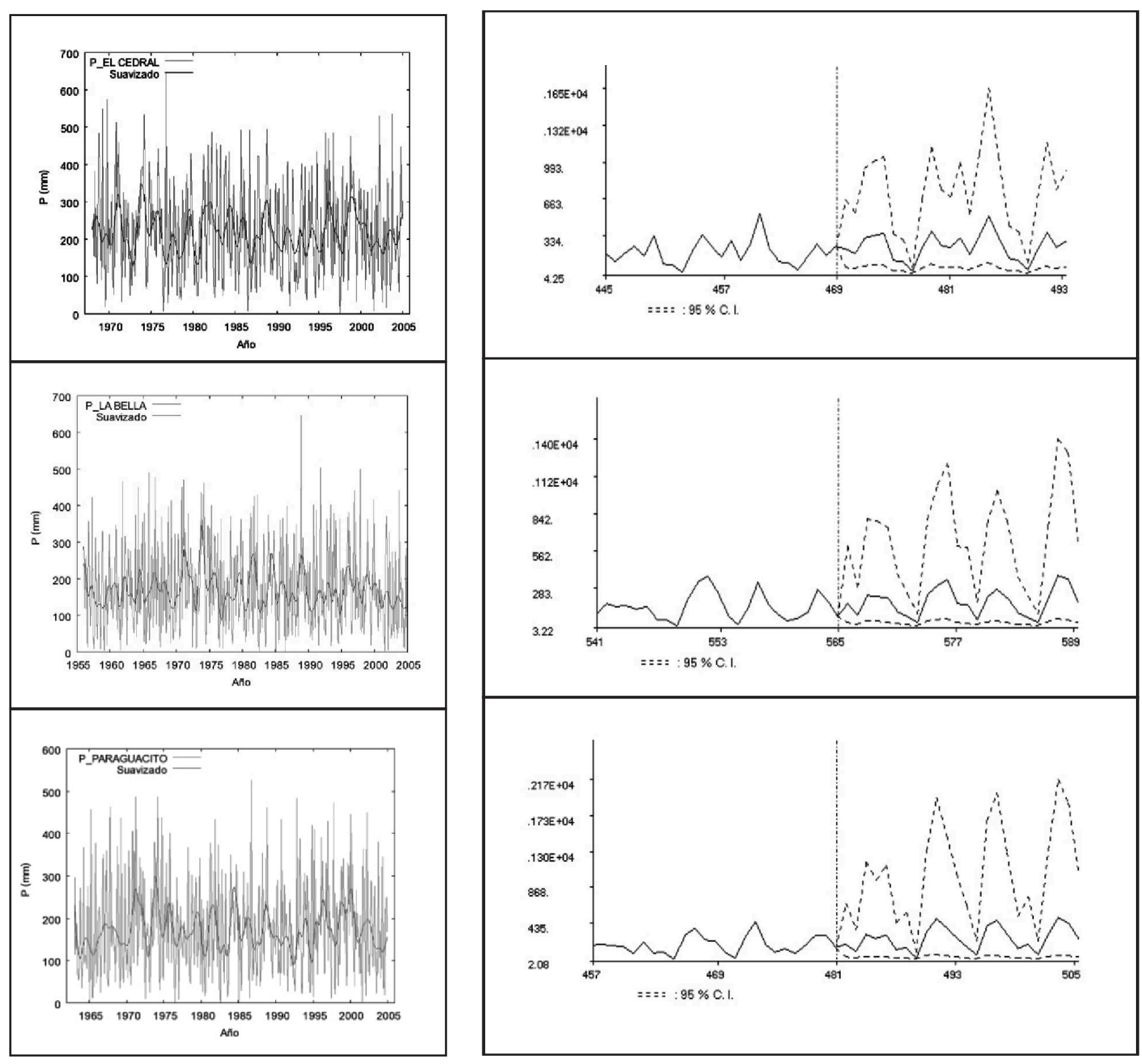

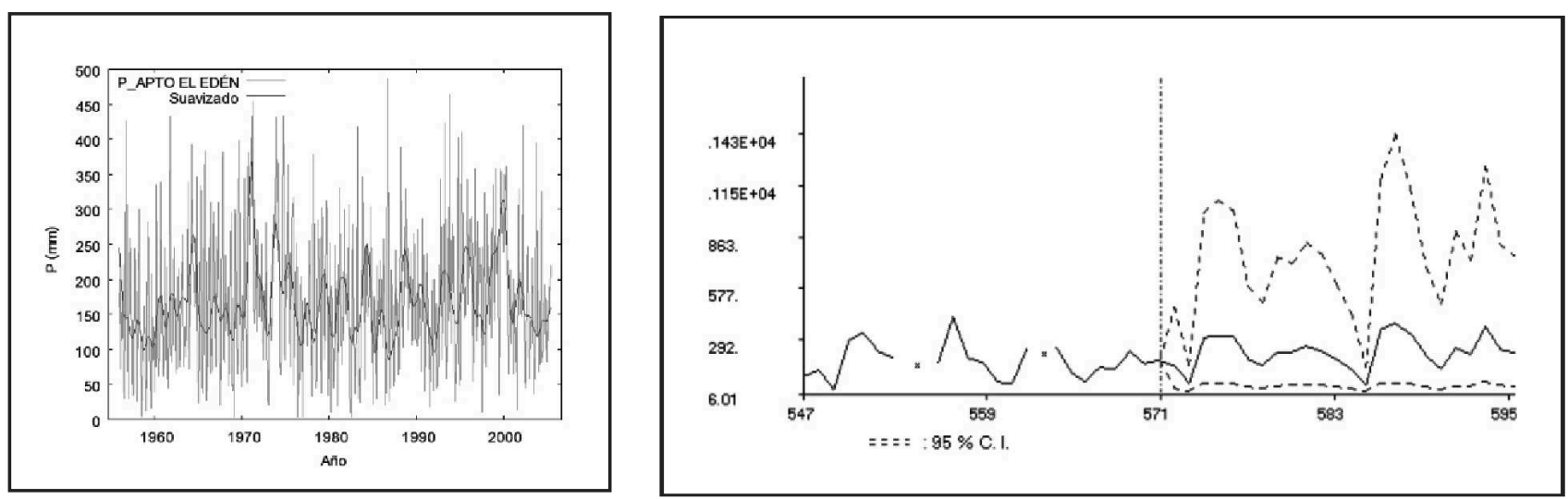

Figura 3 - Series de tiempo para precipitación en mm (García \& otros, 2008)
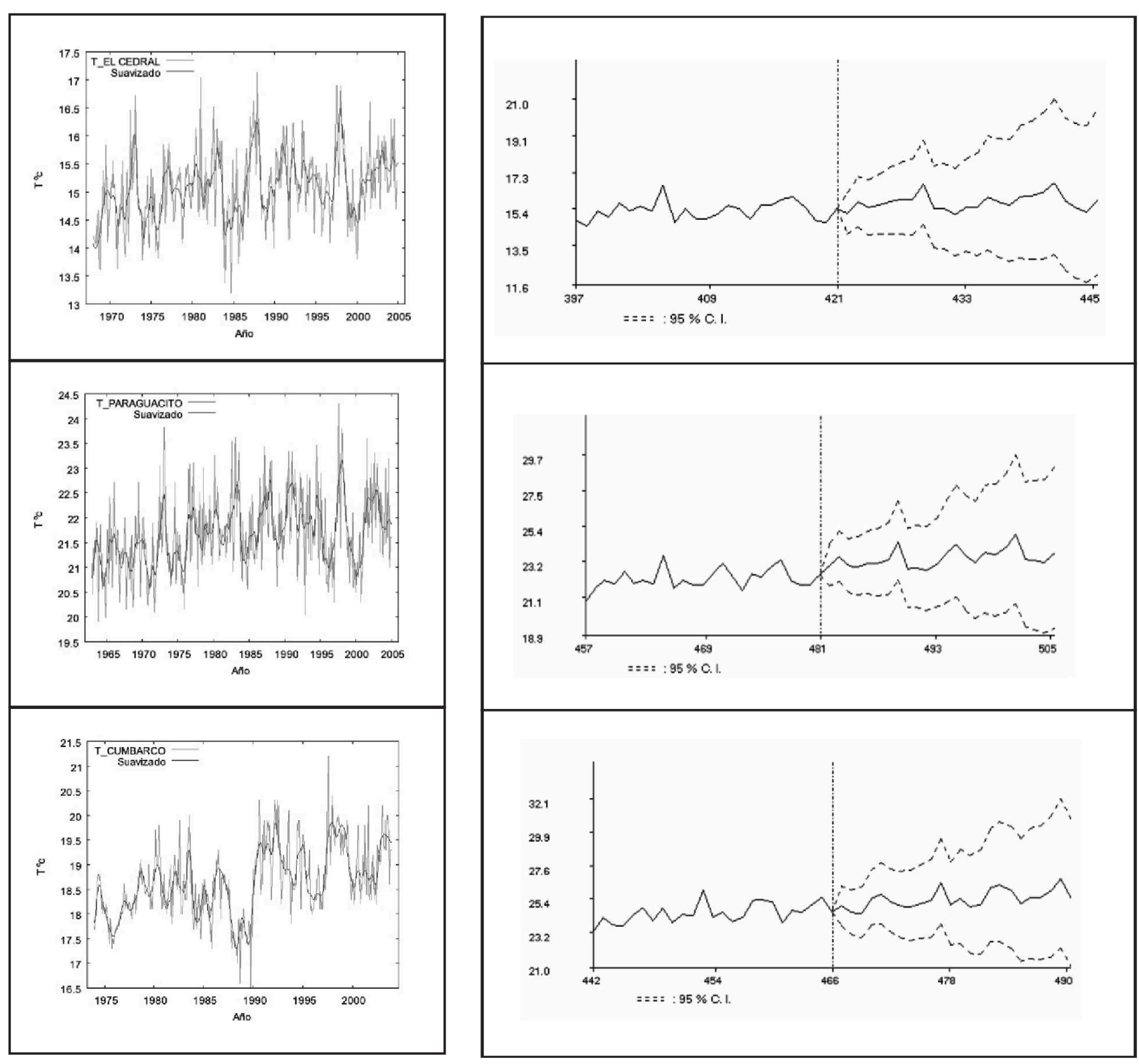

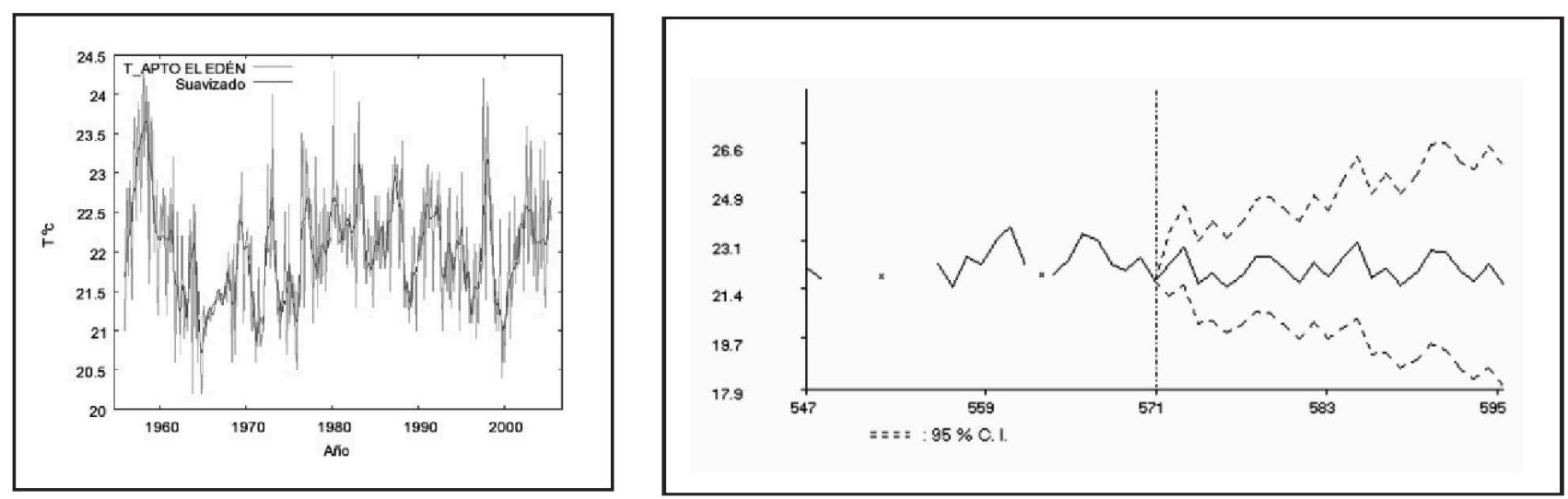

Figura 4 - Series de tiempo para temperatura en ${ }^{\circ} \mathrm{C}$ (García \& otros, 2008)

Para detectar estadísticamente la presencia de tendencia, se realizó una regresión simple de la temperatura en la estación Paraguacito para verificar lo observado en las series de este tipo. Una temperatura al inicio de la serie de $21.27^{\circ} \mathrm{C}$ (1963) y para el año 2013 una temperatura de $22.24^{\circ} \mathrm{C}$, lo que significaría un aumento de casi $1^{\circ} \mathrm{C}$ en un margen de 50 años. Al revisar los resultados obtenidos para éste análisis, se verifica un mal ajuste de la tendencia al presentar un $\mathrm{R}^{2}=$ 0.0949 . Esto podría asociarse a una fuerte variabilidad de los datos que no permiten la determinación acertada de algún tipo de tendencia, tomándose este análisis como insuficiente para la identificación de un aumento en la temperatura.

La mayoría de los modelos analizados se comportan de la forma $\operatorname{ARIMA}(3,1,0)(1,1,0) 12$ pudiendo con ello describir de forma mas certera el comportamiento de la temperatura en esta región.

Las gráficas de las funciones FAS y FAP de los residuales mostraron una barra fuera de los límites de confiabilidad correspondiente al retardo 12 lo cual podría poner en duda la veracidad el modelo, sin embargo, se probó estadísticamente la hipótesis de que las correlaciones entre los errores es cero.

Se cumplió con el criterio de estacionariedad reflejado en los coeficientes dados para cada parámetro de cada modelo ARIMA. Todos se ajustaron correctamente aunque no siendo objeto de estudio la realización de algún pronóstico, se observó un grado aceptable de confianza para la predicción de valores futuros (éstas únicamente se construyeron para corroborar el buen ajuste del modelo ARIMA).

Si bien, no solo las graficas originales observadas en las diferentes estaciones dejan ver una tendencia creciente, si no que también el horizonte de predicción de cada una de ellas trata de indicar un probable incremento en la temperatura a través del tiempo, el cual evidencia de cierta forma un posible cambio climático.

\section{CONCLUSIONES}

Los modelos describieron un comportamiento adecuado con respecto a la serie histórica registrada en la simulación, en la proyección se comportaron con niveles de confianza bajos (temperatura) y niveles de confianza aceptables (precipitación). El análisis del comportamiento de series de tiempo por medio de la metodología Box-Jenkins para la modelación ARIMA ha identificado una estacionalidad cada 12 meses en cada uno de los parámetros en estudio.

Estudios adelantados por el IPCC(2003) exponen una serie de observaciones que se vienen presentando en torno a la temperatura media mundial en la superficie durante el siglo $X X I$, ésta probablemente aumentará a niveles sin precedentes en los últimos 10.000 años. Ahora, con estudios más profundos realizados por expertos en el tema, los resultados se ajustan a la tendencia creciente de los niveles de temperatura, con un nivel de confianza muy bajo explicado por la naturaleza de los datos, hecho que se confirma con los análisis hechos en este trabajo, que parten de registros superiores a los treinta años.

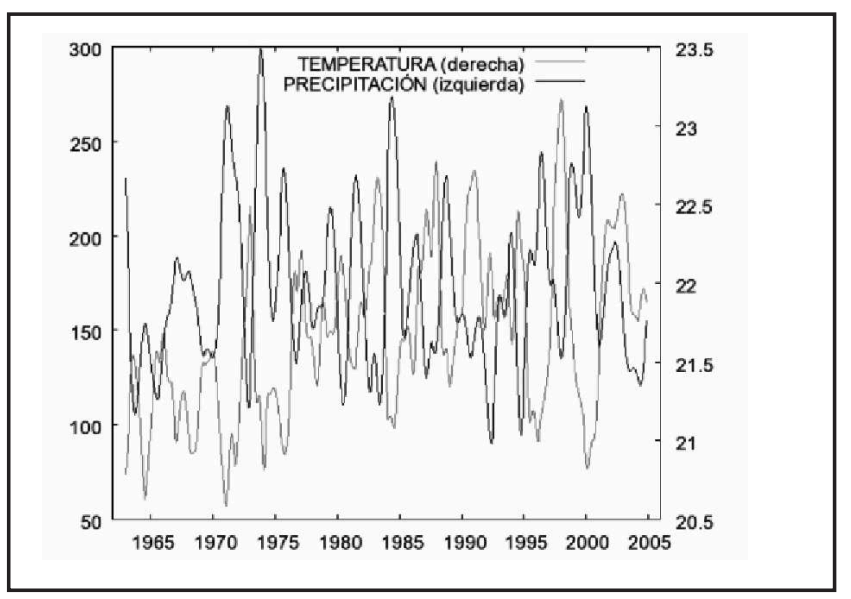

Figura 5 - Serie de tiempo temperatura en ${ }^{\circ} \mathrm{C}$ vs precipitación mm (García \& otros, 2008) 
Al confrontar los valores medios de las series de precipitación y temperatura, en ésta región del país, se observa (Figura 5) cómo los datos registrados de ambas variables son similares inversamente, es decir, cuando la temperatura toma valores altos la precipitación los toma semejantes pero bajos (cuando llueve la temperatura disminuye, en este caso).
Al inicio de ambas gráficas, las series muestran la presencia de variaciones con fluctuaciones fuertes. Entre los años 1975 y 2000 la diferencia visual de las oscilaciones permanece casi constante. Al final de la serie, los datos presentan similitud pero directamente proporcional, marcando una diferencia con lo exhibido por el resto de la gráfica, siendo indicio de un cambio a partir del año 2002.

\section{BIBLIOGRAFÍA}

Arango, S. E. 2003. Análisis de consistencia y complementación de datos faltantes de los registros pluviométricos reportados por la red de medición de la C.R.Q. Trabajo de Grado para optar al titulo de Ingeniero Civil. Programa de Ingeniería Civil. Facultad de Ingeniería. Universidad del Quindío. Armenia.

Box, G. E. P. \& Jenkins, G. M. Time Series Analysis Forecasting and Control. San Francisco: Holder-Day, 1976.

Corporación Autónoma Regional del Quindío. CRQ. 2003. Boletín Meteorológico 2002. Subdirección de Calidad Ambiental. Grupo de Aguas. Armenia.

Corporación Autónoma Regional del Quindío. CRQ. 2004. Plan de Acción Trienal 2004-2006. Armenia.

García R., P. L. 2007. Análisis de la evidencia generada por el cambio climático regional en la cuenca del río Quindío. Centro de Investigación, Desarrollo y Estudio del Recurso Hídrico y el Ambiente, CIDERA. Universidad del Quindío. Armenia.

García R., P. L. Pérez, J. Pedraza, O. E. 2008. La variabilidad climática en la cuenca del Río Quindío. Memorias: XVIII Seminario Nacional de Hidráulica e Hidrología. 14-23. Bogota.

Gobernación del Quindío. 2005. Carta Estadística 2004. Departamento Administrativo de Planeación. Observatorio Económico y Social. Armenia.

Hurtado, L. H., Salcedo, G. E. 1996. Series Temporales con aplicaciones a la Epidemiología y a la Ecología. Universidad del Quindío. Armenia.

Instituto de Hidrología, Meteorología y Estudios Ambientales, IDEAM. Primera Comunicación Nacional. 2001.

Intergovernmental Panel on Climate Change. IPCC. 2003. Cambio Climático 2001: Informe de Síntesis.

Lozano S., G. 2002. Modelo de Simulación de la Calidad del Agua para el Río Quindío. Centro de Investigación, Desarrollo y Estudio del Recurso Hídrico y el Ambiente, CIDERA. Universidad del Quindío. Armenia.

Peláez G., J. A. 2005. Informe Final de Pasantía. Boletín Hidrometeorológico CRQ, 2005. Trabajo de Grado para optar al titulo de Ingeniero Civil. Corporación Autónoma Regional del Quindío. Armenia.

Pérez, J. \& Pedraza, O. E. 2007. Tendencias de variables climatológicas en la subcuenca del río Quindío basados en modelos ARIMA. Trabajo de Grado para optar al titulo de Ingeniero Civil. Centro de Investigación, Desarrollo y Estudio del Recurso Hídrico y el Ambiente, CIDERA. Universidad del Quindío. Armenia. 